\title{
RELAP-7 and PRONGHORN Initial Integration Plan
}

Javier Ortensi

David Andrs

Avery A. Bingham

Richard C. Martineau

John W. Peterson

May 2012

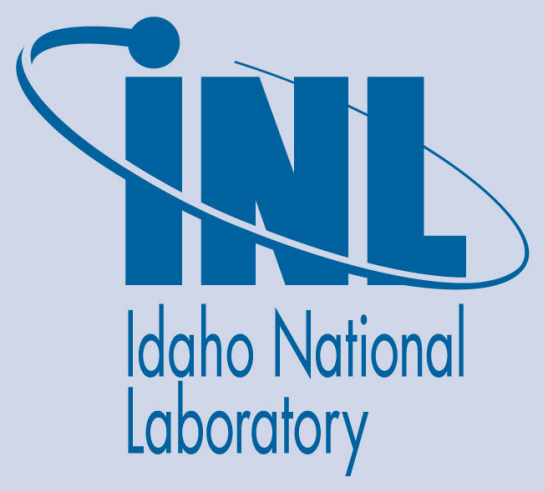

The INL is a U.S. Department of Energy National Laboratory operated by Battelle Energy Alliance 
INL/EXT-12-26016

\title{
RELAP-7 and PRONGHORN Initial Integration Plan
}

\author{
Javier Ortensi \\ David Andrs \\ Avery A. Bingham \\ Richard C. Martineau \\ John W. Peterson \\ May 2012

\section{Idaho National Laboratory \\ Idaho Falls, Idaho 83415} \\ http://www.inl.gov \\ Prepared for the \\ U.S. Department of Energy \\ Office of Nuclear Energy \\ Under DOE Idaho Operations Office \\ Contract DE-AC07-05ID14517
}




\section{INL REPORT}

INL/EXT-12-26016

Unlimited Release

Printed May 2012

\section{RELAP-7 and PRONGHORN Initial Integration Plan}

Javier Ortensi, David Andrs, Avery A Bingham, Richard C Martineau, John W Peterson

Prepared by

Idaho National Laboratory

Idaho Falls, Idaho 83415

The Idaho National Laboratory is a multiprogram laboratory operated by

Battelle Energy Alliance for the United States Department of Energy

under DOE Idaho Operations Office. Contract DE-AC07-05ID14517.

Approved for public release; further dissemination unlimited.

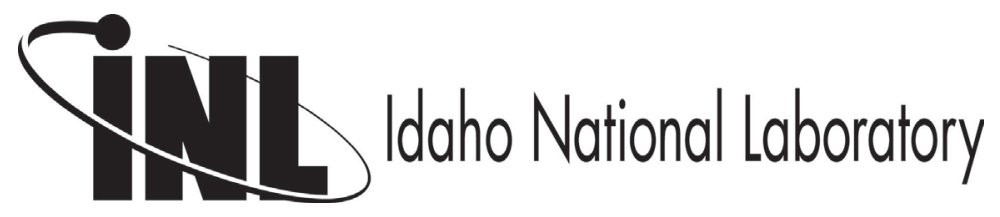


Issued by the Idaho National Laboratory, operated for the United States Department of Energy by Battelle Energy Alliance.

NOTICE: This report was prepared as an account of work sponsored by an agency of the United States Government. Neither the United States Government, nor any agency thereof, nor any of their employees, nor any of their contractors, subcontractors, or their employees, make any warranty, express or implied, or assume any legal liability or responsibility for the accuracy, completeness, or usefulness of any information, apparatus, product, or process disclosed, or represent that its use would not infringe privately owned rights. Reference herein to any specific commercial product, process, or service by trade name, trademark, manufacturer, or otherwise, does not necessarily constitute or imply its endorsement, recommendation, or favoring by the United States Government, any agency thereof, or any of their contractors or subcontractors. The views and opinions expressed herein do not necessarily state or reflect those of the United States Government, any agency thereof, or any of their contractors.

Printed in the United States of America. This report has been reproduced directly from the best available copy.

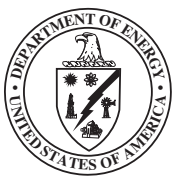


INL/EXT-12-26016

Unlimited Release

Printed May 2012

\section{RELAP-7 and PRONGHORN Initial Integration Plan}

\author{
Javier Ortensi \\ Reactor Physics Analysis and Design \\ Idaho National Laboratory \\ P.O. Box 1625 \\ Idaho Falls, ID 83415-3840 \\ Javier.Ortensi@inl.gov
}

Avery A Bingham

Fuel Modeling and Simulation

Idaho National Laboratory

P.O. Box 1625

Idaho Falls, ID 83415-3840

Avery.Bingham@inl.gov
David Andrs

Fuel Modeling and Simulation

Idaho National Laboratory

P.O. Box 1625

Idaho Falls, ID 83415-3840

David.Andrs@inl.gov

Richard C Martineau

Fuel Modeling and Simulation

Idaho National Laboratory

P.O. Box 1625

Idaho Falls, ID 83415-3840

Richard.Martineau@inl.gov

John W Peterson

Fuel Modeling and Simulation

Idaho National Laboratory

P.O. Box 1625

Idaho Falls, ID 83415-3840

JW.Peterson@inl.gov 


\begin{abstract}
Modern nuclear reactor safety codes require the ability to solve detailed coupled neutronicthermal fluids problems. For larger cores, this implies fully coupled 3-D spatial dynamics with appropriate feedback models that can provide enough resolution to accurately compute core heat generation and removal during steady and unsteady conditions. The reactor analysis code PRONGHORN is being coupled to RELAP-7 as a first step to extend RELAP's current capabilities. This report details the mathematical models, the type of coupling, and the testing that will be used to produce an integrated system. RELAP-7 is a MOOSE-based application that solves the continuity, momentum, and energy equations in 1-D for a compressible fluid. The pipe and joint capabilities enable it to model parts of the power conversion unit. The PRONGHORN application, also developed on the MOOSE infrastructure, solves the coupled equations that define the neutron diffusion, fluid flow, and heat transfer in a 3-D core model. Initially, the two systems will be loosely coupled to simplify the transition towards a more complex infrastructure. The integration will be tested with the OECD/NEA MHTGR-350 Coupled Neutronics-Thermal Fluids benchmark model.
\end{abstract}




\section{Contents}

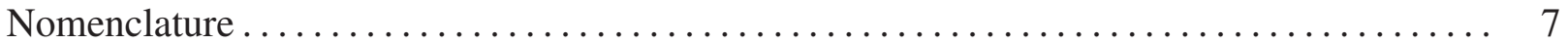

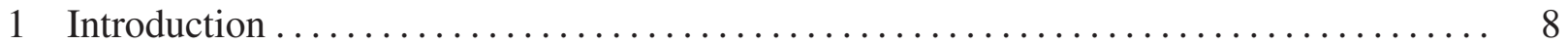

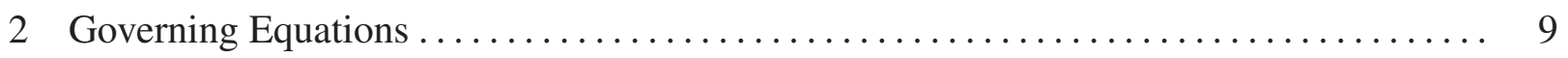

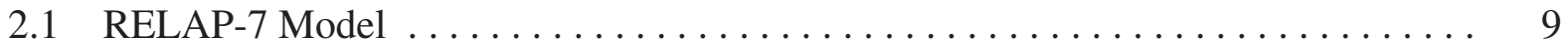

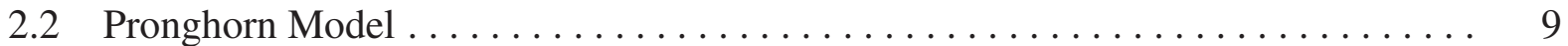

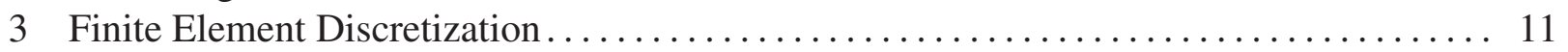

$3.1 \quad$ RELAP-7 Model . . . . . . . . . . . . . . . . . . . . . . . . . . 11

3.2 Pronghorn Model ...................................... 12

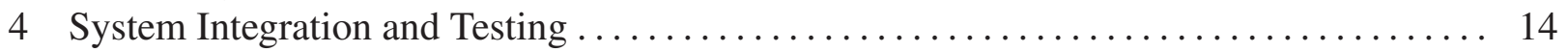

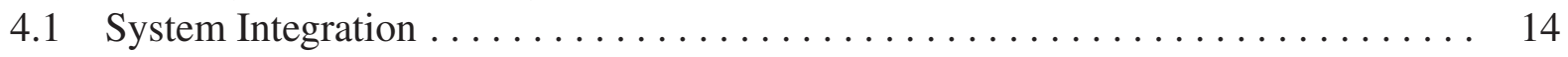

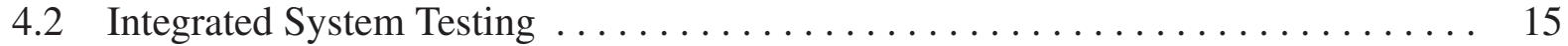

References.................................................... 19 


\section{Figures}

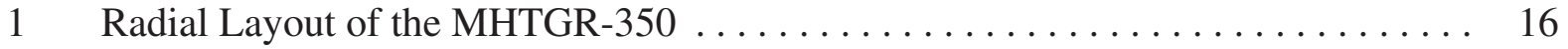

2 Axial Layout of the MHTGR-350 . . . . . . . . . . . . . . . . . . . . . 17

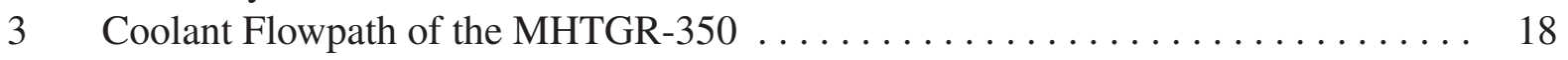

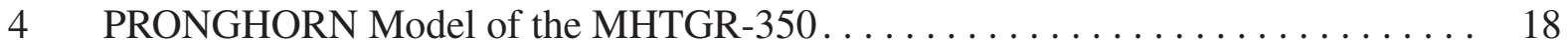




\section{Nomenclature}

$\varepsilon=$ material porosity

$\rho_{f}=$ density of the fluid

$\mu=$ dynamic viscosity of the fluid

$K=$ permeability

$P=$ static pressure

$W=$ isotropic friction coefficient

$\tilde{W}=$ effective friction coefficient tensor

$\vec{g}=$ gravity

$\vec{u}=$ intrinsic phase averaged velocity vector

$T=$ temperature

$R=$ universal gas constant

$\langle f, \Psi\rangle_{\Gamma}=$ surface integral of $f \Psi$

$(\vec{f}, \nabla \Psi)=$ volume integral of $\vec{f} \cdot \nabla \Psi$ over the whole domain

$d_{h}=$ hydraulic diameter

$\hat{n}_{x}=$ the $x$-component of the "outward unit normal" of the domain 


\section{Introduction}

The RELAP-7 code is part of the next generation of nuclear reactor system safety analysis codes being developed at the Idaho National Laboratory (INL). The current capabilities of RELAP-7 include single phase fluid flow in 1-D pipes and joints. The pipe is the fundamental component of the RELAP-7 code, and all problems modeled with RELAP-7 consist of networks of these pipes. For non-isothermal cases, three equations are solved: continuity, momentum and energy equations (continuity and momentum only, if isothermal). Wall friction factors and convective heat transfer coefficients are calculated through closure laws or provided by user inputs. The effect of gravity is taken into account through pipe orientation and flow direction. The latest model includes heat removal from a fixed power distribution [1]. However, the current version of RELAP-7 does not contain a nuclear reactor module to perform the necessary coupled core power generation and heat removal computations. The next step in the evolution of the code is the addition of thermal-fluid feedback from a full core coupled neutronics-thermal fluids solver.

The PRONGHORN code, which was initially developed to model gas cooled pebble bed reactors [2], is being extended for the modeling of prismatic high temperature reactors. The code solves the neutron diffusion equation, with a Darcy fluid flow model, and a conjugate heat transfer model for solid and fluid energy transfer. Some of the improvements in PRONGHORN include an extension to 3-D geometry and the use of a homogenized stationary two-phase flow model (extended HazenDepuit-Darcy).

Both RELAP-7 and PRONGHORN are applications developed on the MOOSE system. MOOSE (Multiphysics Object-Oriented Simulation Environment) is a framework for solving computational engineering problems in a well-planned, managed, and coordinated way. By leveraging open source software packages, such as PETSC (a nonlinear solver developed at Argonne National Laboratory) and LibMesh (a Finite Element Analysis package developed at University of Texas), MOOSE significantly reduces the expense and time required to develop new applications. Numerical integration methods and mesh management for parallel computation are provided by MOOSE. Therefore, RELAP-7 and PRONGHORN code developers only need to focus on physics and user experience. By using the MOOSE development framework, the RELAP-7 and PRONGHORN codes can take advantage of all contributions made to MOOSE by other application developers which follows the paradigm for efficient modern software design. Multiphysics and multiple dimensional analyses capabilities can be obtained by coupling RELAP-7 and other MOOSE based applications and by leveraging with capabilities developed by other DOE programs such as PRONGHORN. These capabilities strengthen the focus of RELAP-7 to systems analysis-type simulations and gives priority to retain and significantly extend RELAP5s capabilities. 


\section{Governing Equations}

\subsection{RELAP-7 Model}

A simplified flow model for compressible flow is used in this work as an initial step towards more complex models. The equations for an non-isothermal fluid in a one-dimensional pipe are

\section{- Fluid Continuity Equation}

$$
\frac{\partial \rho}{\partial t}+\frac{\partial(\rho u)}{\partial x}=0
$$

- Momentum Equation

$$
\frac{\partial(\rho u)}{\partial t}+\frac{\partial\left(\rho u^{2}+P_{\mathrm{EOS}}\right)}{\partial x}-\rho g_{x}+f \frac{\rho}{2 d_{h}}|u| u=0
$$

where $g_{x}$ is the component of the gravity vector in the $x$-direction and $f$ is a dimensionless friction factor. An equation of state of the general form $P_{\mathrm{EOS}}=P(\rho, \rho u)$ completes the definition of the model.

- Energy Conservation Equation

$$
\frac{\partial(\rho E)}{\partial t}+\frac{\partial(\rho u H)}{\partial x}+H_{w} a_{w}\left(T-T_{w}\right)+u\left(f \frac{\rho}{2 d_{h}}|u| u-\rho g_{x}\right)=0
$$

where $H \equiv E+\frac{P_{\mathrm{EOS}}}{\rho}$ is the total enthalpy, and $H_{w}, a_{w}$ are generic heat-transfer coefficients intended to model heat loss/addition along the length of the one-dimensional pipe. The total energy $E \equiv e+\frac{u^{2}}{2}$, where $e$ is the internal energy of the fluid. Equations of state of the form $P_{\mathrm{EOS}}=P(\rho, e)$ and $T=T(\rho, e)$ are required to close the system.

\subsection{Pronghorn Model}

The Pronghorn multi-physics equation set includes: 1) a Darcy fluid flow model, 2) a conjugate model for heat transfer, and 3) a neutron diffusion model for neutron interactions.

\section{- Fluid Continuity Equation}

The conservation of fluid mass with no phase change and a single fluid species in a homogenized porous medium is expressed by [3]

$$
\frac{\partial \varepsilon \rho_{f}}{\partial t}+\nabla \cdot\left(\varepsilon \rho_{f} \vec{u}\right)=0
$$




\section{- Momentum Equation}

The governing equation for momentum in the Darcy flow model with a gravitational term is

$$
\varepsilon \nabla P-\varepsilon \rho_{f} \vec{g}+W \rho_{f} \vec{u}=0,
$$

\section{- Pressure Poisson Equation}

The governing equation for pressure in the Darcy flow model is obtained by solving Eq. (5) for $\rho_{f} \vec{u}$ and substituting into Eq. (4):

$$
\frac{\partial \varepsilon \rho_{f}}{\partial t}+\nabla \cdot\left(\frac{\varepsilon^{2}}{W}\left(-\nabla P+\rho_{f} \vec{g}\right)\right)=0
$$

The ideal gas law is then used to substitute the gas density and obtain an equation in terms of pressure. The resulting equation is a special form of the pressure Poisson equation

$$
\frac{1}{R} \frac{\partial}{\partial t}\left(\frac{\varepsilon P}{T_{f}}\right)-\nabla \cdot \frac{\varepsilon^{2}}{W} \nabla P+\nabla \cdot \frac{\varepsilon^{2}}{W R} \frac{P \vec{g}}{T_{f}}=0 .
$$

\section{- Fluid Energy Equation}

The governing equations for heat transfer in the fluid:

$$
\frac{\partial}{\partial t}\left[\varepsilon \rho_{f} c_{p f} T_{f}\right]+\nabla \cdot\left(\varepsilon \rho_{f} c_{p f} \vec{u} T_{f}\right)-\nabla \cdot \varepsilon \kappa_{f} \nabla T_{f}+\alpha\left(T_{f}-T_{s}\right)=0,
$$

- Solid Energy Equation

The governing equation for heat transfer in the solid medium

$$
\frac{\partial}{\partial t}\left[(1-\varepsilon) \rho_{s} c_{p s} T_{s}\right]-\nabla \cdot\left(\kappa_{s, \mathrm{eff}} \nabla T_{s}\right)+\alpha\left(T_{s}-T_{f}\right)-Q=0,
$$

\section{- Neutron Diffusion Equation}

The governing equations for neutron diffusion:

$$
\frac{1}{v_{g}} \frac{\partial \phi_{g}}{\partial t}-\nabla \cdot D_{g} \nabla \phi_{g}+\Sigma_{R g} \phi_{g}-Q_{g}=0
$$

where

$$
Q_{g}=-(1-\beta) \chi_{g} \sum_{g^{\prime}} v \Sigma_{f g^{\prime}} \phi_{g^{\prime}}-\sum_{g^{\prime}, g^{\prime} \neq g} \Sigma_{s}^{g^{\prime} \rightarrow g} \phi_{g^{\prime}}-\sum_{k} \chi_{d_{g, k}} \lambda_{k} C_{k}
$$

\section{- Delayed Neutron Precursor Equation}

The governing equation for the $k^{\text {th }}$ delayed neutron precursor

$$
\frac{\partial C_{k}}{\partial t}+\lambda_{k} C_{k}-\sum_{g^{\prime}} \beta_{k, g^{\prime}} v \Sigma_{f g^{\prime}} \phi_{g^{\prime}}=0
$$




\section{Finite Element Discretization}

The method of weighted residuals (or Galerkin procedure) is used to obtain the approximation to the integral form of the PDEs. The weighted residual form of the equations is derived by multiplying each term of the differential equations by a test function $\Psi$ and integrating over the volume. The final weak formulation is obtained by trading differentiation with the product rule. The method of weighted residuals is used to formulate a nonlinear system of the form

$$
\mathbf{F}(\mathbf{U})=0 .
$$

This system is then solved iteratively via the JFNK method [4, 5]. The JFNK method is a combination of the quadratically convergent Newton method and a Krylov subspace iterative method. The first-order Taylor expansion of Eq. (12) about $\mathbf{U}^{m}$ gives the following linear system,

$$
\mathbb{J}^{m} \delta \mathbf{U}^{m}=-\mathbf{F}\left(\mathbf{U}^{m}\right),
$$

where $\mathbb{J}_{i, j}^{m} \equiv \frac{\partial \mathbf{F}_{i}\left(\mathbf{U}^{m}\right)}{\partial \mathbf{U}_{j}^{m}}$ is the $i, j$ element of Jacobian matrix for the $m^{t h}$ Newton iteration. The linear system (13) is solved using a Krylov subspace method and the solution is updated as

$$
\mathbf{U}^{m+1}=\mathbf{U}^{m}+d \delta \mathbf{U}^{m}
$$

where $0<d \leq 1$ is a scalar damping parameter chosen adaptively to avoid unphysical solutions.

Because Krylov methods merely require matrix-vector products, a Jacobian-Free Newton-Krylov (JFNK) method can be used to alleviate the explicit formation of the expensive Jacobian matrix. In JFNK the matrix-vector product is approximated by the finite difference form,

$$
\mathbb{J} \mathbf{v} \approx \frac{\mathbf{F}(\mathbf{U}+h \mathbf{v})-\mathbf{F}(\mathbf{U})}{h},
$$

where $h$ is the perturbation parameter and $\mathbf{v}$ is provided by the Krylov method.

\subsection{RELAP-7 Model}

The weak formulation that is associated with (1), (2), and (3) leads to the following nonlinear residual functions:

- Fluid Continuity Nonlinear Residual Function

$$
\mathbf{F}_{\rho}(\boldsymbol{U})=\left(\frac{\partial \rho}{\partial t}, \Psi\right)-\left(\rho u, \frac{\partial \Psi}{\partial x}\right)+\left\langle\rho u \hat{n}_{x}, \Psi\right\rangle_{\Gamma}
$$

- Momentum Nonlinear Residual Function

$$
\begin{aligned}
\mathbf{F}_{\rho u}(\boldsymbol{U}) & =\left(\frac{\partial(\rho u)}{\partial t}, \Psi\right)-\left(\rho g_{x}, \Psi\right)+\left(f \frac{\rho}{2 d_{h}}|u| u, \Psi\right)-\left(\rho u^{2}, \frac{\partial \Psi}{\partial x}\right)+\left(P, \frac{\partial \Psi}{\partial x}\right) \\
& +\left\langle\rho u^{2} \hat{n}_{x}, \Psi\right\rangle_{\Gamma}+\left\langle P \hat{n}_{x}, \Psi\right\rangle_{\Gamma}
\end{aligned}
$$




\section{- Energy Conservation Nonlinear Residual Function}

Substituting $\rho u H=\rho u\left(E+\frac{P}{\rho}\right)=u(\rho E+P)$ in (3) and applying the Galerkin procedure yields

$$
\begin{aligned}
\mathbf{F}_{\rho E}(\boldsymbol{U})= & \left(\frac{\partial(\rho E)}{\partial t}, \Psi\right)+\left(H_{w} a_{w}\left(T-T_{w}\right), \Psi\right)+\left(f \frac{\rho}{2 d_{h}}|u| u^{2}, \Psi\right)-\left(u \rho g_{x}, \Psi\right) \\
& -\left(\rho u E, \frac{\partial \Psi}{\partial x}\right)-\left(u P, \frac{\partial \Psi}{\partial x}\right)+\left\langle\rho u E \hat{n}_{x}, \Psi\right\rangle_{\Gamma}+\left\langle u P \hat{n}_{x}, \Psi\right\rangle_{\Gamma}
\end{aligned}
$$

where

$$
\begin{aligned}
& \mathbf{F}=\text { nonlinear residual function } \\
& \mathbf{U}=(\rho, \rho u, \rho E)^{T}
\end{aligned}
$$

$\Psi$ is a scalar test function

\subsection{Pronghorn Model}

The weak formulation that is associated with the Pronghorn equation set is obtained by applying the method of weighted residuals to equations (5), (7), (8), (9), (10), and (11), respectively.

- Momentum Nonlinear Residual Function

$$
\mathbf{F}_{\rho \vec{u}}(\boldsymbol{U})=(\varepsilon \nabla P, \Psi)-\left(\varepsilon \rho_{f} \vec{g}, \Psi\right)+\left(W \rho_{f} \vec{u}, \Psi\right)
$$

- Pressure Poisson Nonlinear Residual Function

$$
\begin{aligned}
\mathbf{F}_{P}(\boldsymbol{U}) & =\left(\frac{\partial}{\partial t}\left(\frac{\varepsilon P}{R T_{f}}\right), \Psi\right)+\left(\frac{\varepsilon^{2}}{W} \nabla P, \nabla \Psi\right)-\left(\frac{\varepsilon^{2}}{W} \rho_{f} \vec{g}, \nabla \Psi\right) \\
& -\left\langle\frac{\varepsilon^{2}}{W} \nabla P, \Psi\right\rangle+\left\langle\frac{\varepsilon^{2}}{W} \rho_{f} \vec{g}, \Psi\right\rangle
\end{aligned}
$$

- Fluid Energy Nonlinear Residual Function

$$
\begin{aligned}
\mathbf{F}_{T_{f}}(\boldsymbol{U}) & =\left(\frac{\partial \varepsilon \rho_{f} c_{p f} T_{f}}{\partial t}, \Psi\right)-\left(\varepsilon \rho_{f} c_{p f} \vec{u} T_{f}, \nabla \Psi\right)+\left(\varepsilon \kappa_{f} \nabla T_{f}, \nabla \Psi\right) \\
& +\left(\alpha\left(T_{f}-T_{s}\right), \Psi\right)+\left\langle\varepsilon \rho_{f} c_{p f} \vec{u} T_{f}, \Psi\right\rangle-\left\langle\varepsilon \kappa_{f} \nabla T_{f}, \Psi\right\rangle
\end{aligned}
$$

- Solid Energy Nonlinear Residual Function

$$
\begin{aligned}
\mathbf{F}_{T_{s}}(\boldsymbol{U}) & =\left(\frac{\partial(1-\varepsilon) \rho_{s} c_{p s} T_{s}}{\partial t}, \Psi\right)+\left(\kappa_{s e f f} \nabla T_{s}, \nabla \Psi\right) \\
& +\left(\alpha\left(T_{s}-T_{f}\right), \Psi\right)-(Q, \Psi)-\left\langle\kappa_{\text {seff }} \nabla T_{s}, \Psi\right\rangle
\end{aligned}
$$


- Neutron Diffusion Nonlinear Residual Function

$$
\begin{aligned}
\mathbf{F}_{\phi_{g}}(\boldsymbol{U}) & =\left(\frac{1}{v_{g}} \frac{\partial \phi_{g}}{\partial t}, \Psi\right)+\left(D_{g} \nabla \phi_{g}, \nabla \Psi\right)-\left\langle D_{g} \nabla \phi_{g}, \Psi\right\rangle+\left(\Sigma_{r} \phi_{g}, \Psi\right) \\
& -\left((1-\beta) \chi_{g} \sum_{g^{\prime}} v \Sigma_{f g^{\prime}} \phi_{g^{\prime}}, \Psi\right)-\left(\sum_{g^{\prime}, g^{\prime} \neq g} \Sigma_{s}^{g^{\prime} \rightarrow g} \phi_{g^{\prime}}, \Psi\right)-\left(\sum_{k} \chi_{d_{g, k}} \lambda_{k} C_{k}, \Psi\right)
\end{aligned}
$$

- Delayed Neutron Precursor Nonlinear Residual Function

$$
\mathbf{F}_{C_{k}}(\boldsymbol{U})=\left(\frac{\partial C_{k}}{\partial t}, \Psi\right)+\left(\lambda_{k} C_{k}, \Psi\right)-\left(\sum_{g^{\prime}} \beta_{k, g^{\prime}} v \Sigma_{f g^{\prime}} \phi_{g^{\prime}}, \Psi\right)
$$

where

$$
\begin{aligned}
& \mathbf{F}=\text { nonlinear residual function } \\
& \mathbf{U}=\left(\rho \vec{u}, P, T_{f}, T_{s}, \phi_{g}, C_{k}\right)^{T}
\end{aligned}
$$

$\Psi$ is a scalar test function 


\section{System Integration and Testing}

\subsection{System Integration}

The integration of RELAP-7 and PRONGHORN will initially be based on a loose coupling of the system, whereby each application will use an independent mesh. A data exchange will be implemented to couple the codes. This type of direct coupling has been successfully used in the past with the TINTE time dependent core solver and the Flownex systems code [6].To determine the required parameters for the exchange we will look at the fluid boundary terms.

For the RELAP-7 model the coupling arises from the boundary terms of the fluid continuity, momentum, and fluid energy equations

$$
\begin{aligned}
& \left\langle\rho u \hat{n}_{x}, \Psi\right\rangle_{\Gamma} \\
& \left\langle\rho u^{2} \hat{n}_{x}, \Psi\right\rangle_{\Gamma}+\left\langle P \hat{n}_{x}, \Psi\right\rangle_{\Gamma} \\
& \left\langle\rho u E \hat{n}_{x}, \Psi\right\rangle_{\Gamma}+\left\langle u P \hat{n}_{x}, \Psi\right\rangle_{\Gamma}
\end{aligned}
$$

The corresponding boundary conditions for the RELAP-7 fluid medium are expressed as

$$
\begin{array}{ll}
P=P_{P H_{\text {inlet }}} & \in \Gamma_{R 7_{\text {outlet }}} \\
\vec{n} \cdot\left[-\frac{\varepsilon}{W} \nabla P+\frac{\varepsilon \rho_{f} \vec{g}}{W}\right]=(\vec{n} \cdot \rho \vec{u})_{P H_{\text {outlet }}} & \in \Gamma_{R 7_{\text {inlet }}} \\
T_{f}=T_{P H_{\text {outlet }}} & \in \Gamma_{R 7_{\text {inlet }}}
\end{array}
$$

where

$P_{P H_{\text {inlet }}}$ is the inlet pressure pressure computed in PRONGHORN that will be imposed on the RELAP-7 outlet boundary, $\Gamma_{R 7_{\text {outlet }}}$

a fixed, uniform mass flow rate condition is set at the inlet boundary, $\Gamma_{R 7_{\text {inlet }}}$

a fixed, uniform temperature from the PRONGHORN model, $T_{P H_{\text {outlet }}}$, is set at the RELAP-7 inlet boundary, $\Gamma_{R 7_{\text {inlet }}}$

For the PRONGHORN model the coupling arises from the boundary terms of the fluid pressure and fluid energy equations

$$
\begin{aligned}
& \left\langle\frac{\varepsilon^{2}}{W} \nabla P, \Psi\right\rangle+\left\langle\frac{\varepsilon^{2}}{W} \rho_{f} \vec{g}, \Psi\right\rangle \\
& \left\langle\varepsilon \rho_{f} c_{p f} \vec{u} T_{f}, \Psi\right\rangle-\left\langle\varepsilon \kappa_{f} \nabla T_{f}, \Psi\right\rangle
\end{aligned}
$$


Note that the momentum equation in the PRONGHORN model does not contain any boundary terms. The conditions for this equation are imposed via the pressure Poisson equation. The corresponding boundary conditions for the fluid medium are expressed as

$$
\begin{array}{ll}
P=P_{R 7_{\text {inlet }}} & \in \Gamma_{P H_{\text {outlet }}} \\
\vec{n} \cdot\left[-\frac{\varepsilon}{W} \nabla P+\frac{\varepsilon \rho_{f} \vec{g}}{W}\right]=(\vec{n} \cdot \rho \vec{u})_{R 7_{\text {outlet }}} & \in \Gamma_{P H_{\text {inlet }}} \\
T_{f}=T_{R 7_{\text {outlet }}} & \in \Gamma_{P H_{\text {inlet }}}
\end{array}
$$

where

$P_{R 7_{\text {inlet }}}$ is the inlet pressure computed in RELAP-7 that will be imposed on the PRONGHORN outlet boundary, $\Gamma_{P H_{\text {outlet }}}$

a fixed, uniform mass flow rate condition is set at the inlet boundary, $\Gamma_{P H_{\text {inlet }}}$

a fixed, uniform temperature from RELAP-7 model, $T_{R 7_{\text {out }}}$, is set at the inlet boundary, $\Gamma_{P H_{\text {inlet }}}$

In order to successfully couple the applications, both fluid heat removal and compression stages will need to be modeled in the RELAP-7 1-D pipe model. This can be done in a number of ways with pipe models to achieve the desired outlet conditions. The main difficulty with this coupling will be the correct adjustment of the heat removal and compression stage to ensure a stable system. Other difficulties might arise for non-steady problems, since both physics will need to be converged before performing a time step advancement.

\subsection{Integrated System Testing}

The General Atomics MHTGR-350 $\mathrm{MW}$ th design will be used in the initial coupling of the codes. The detailed design is based on the OECD/NEA Coupled Neutronics-Thermal Fluids Benchmark [7]. The benchmark provides a full specification including a set of temperature dependent cross sections and thermophysical properties that will ease the task of preparing the necessary data to couple the codes.

The radial layout of the MHTGR-350 model is shown in Figure 1. From the center radially to the periphery, the core includes an inner reflector region (grey), annular active core (orange and red), replaceable reflector (grey), and permanent reflector ( $\tan$ ) regions. The axial layout is included in Figure 2 and shows, from top to bottom, the upper plenum, core restraint element, upper reflector, active core, lower reflector, and lower plenum regions. The flow in the reactor is depicted in Figure 3. The coolant flows through the outer shell of the cross vessel and enters the reactor pressure vessel cooling the metallic core support structure. The flow continues up the coolant channels and mixes in the upper plenum, where is then directed into the top reflector and active core regions. After removing the heat from the core region the coolant passes through the bottom transition 
reflector and flow distribution blocks. Finally, the flow mixes in the lower plenum and exits the core through the inner shell of the cross vessel.

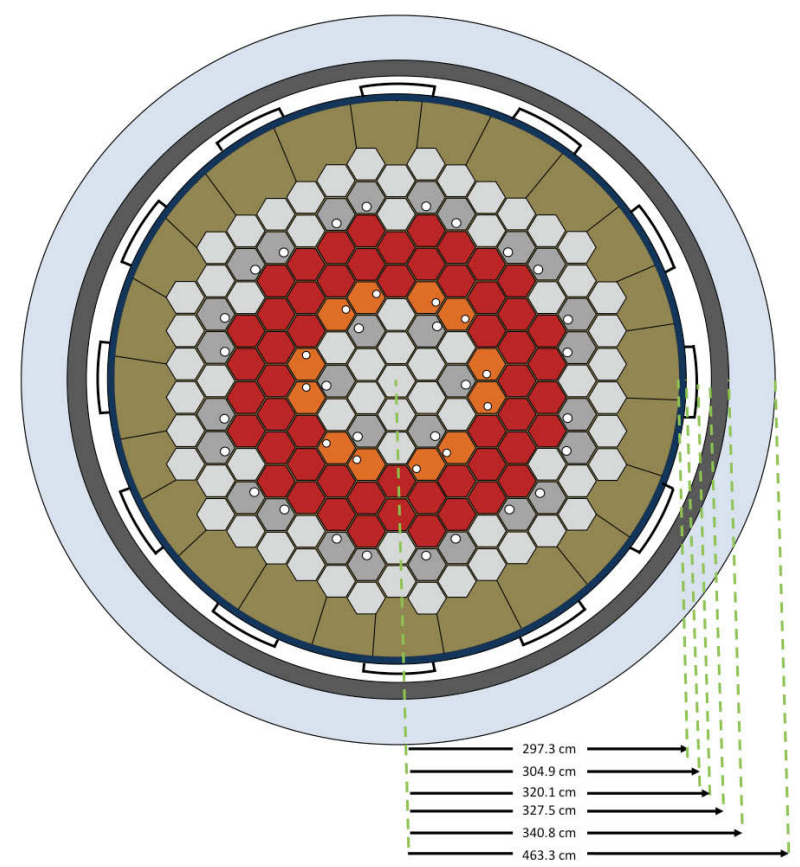

Figure 1: Radial Layout of the MHTGR-350

The PRONGHORN geometry will entail $1 / 3^{\text {rd }}$ core symmetry in 3-D of the MHTGR-350, shown in Figure 4. The figure includes the 34 cross section regions, including the control rod bank that is detailed in the radial detail. The direction of the flow is simplified to include only flow from the top to the bottom of the core. Therefore, the inlet conditions will be set at the upper plenum and the outlet conditions at the lower plenum. The modeling of the riser flow channels is omitted from this test. The steady state neutron diffusion equation will be solved for 26 groups. Non-local heating effects and radiation heat transfer will be neglected.

The initial simulation will entail the approach to steady state condition (null transient). In this type of calculation the xenon buildup is tracked or directly calculated to achieve the steady state condition. In the MHTGR model, the 220 burnup regions of the core have different powers and, consequently, different xenon concentrations. The cross sections for the benchmark include the effects of the variations in fuel and moderator temperature, as well as xenon concentrations. The tracked parameters include: system pressure, core inlet temperature, core outlet temperature, core fuel temperature distribution, core moderator temperature distribution, core fluid temperature distribution, xenon concentration distribution, core power distribution, and local static pressure distribution. 


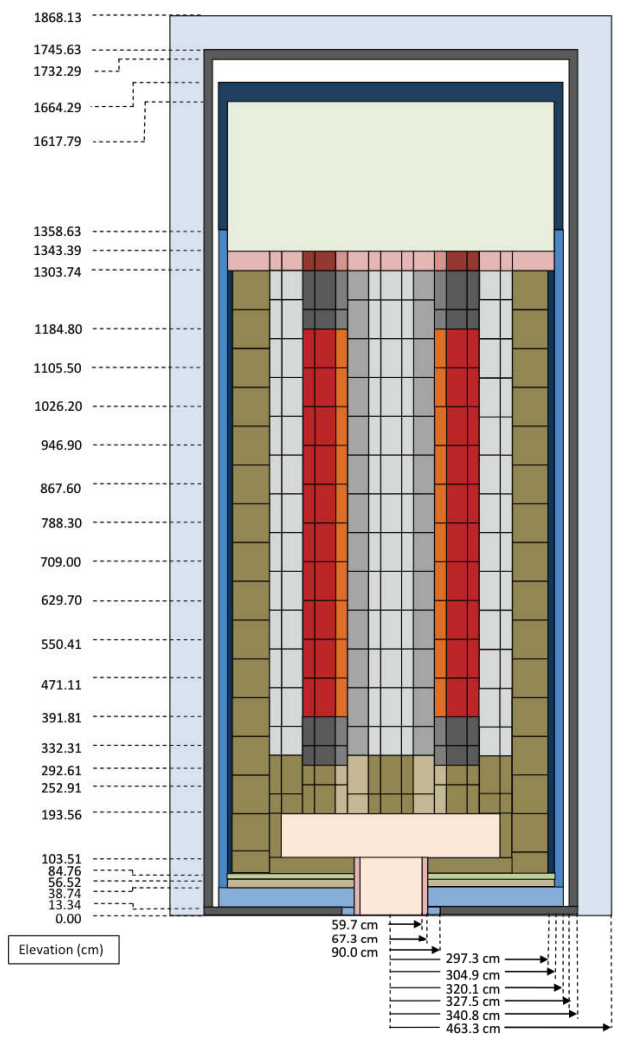

Figure 2: Axial Layout of the MHTGR-350 


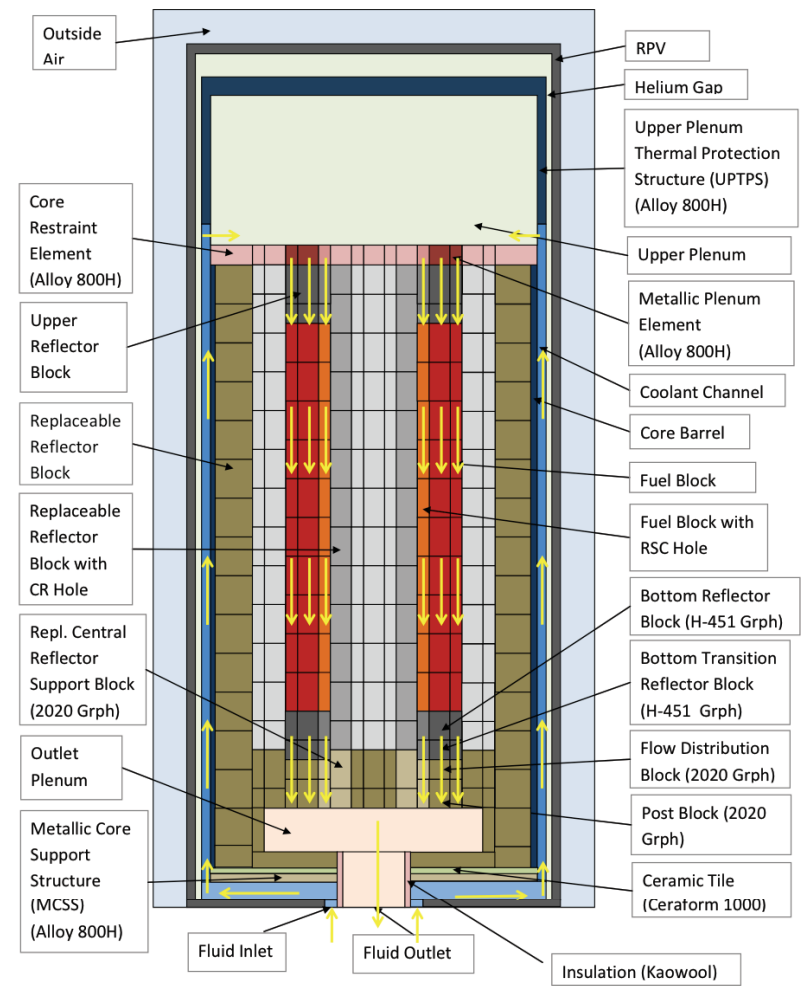

Figure 3: Coolant Flowpath of the MHTGR-350

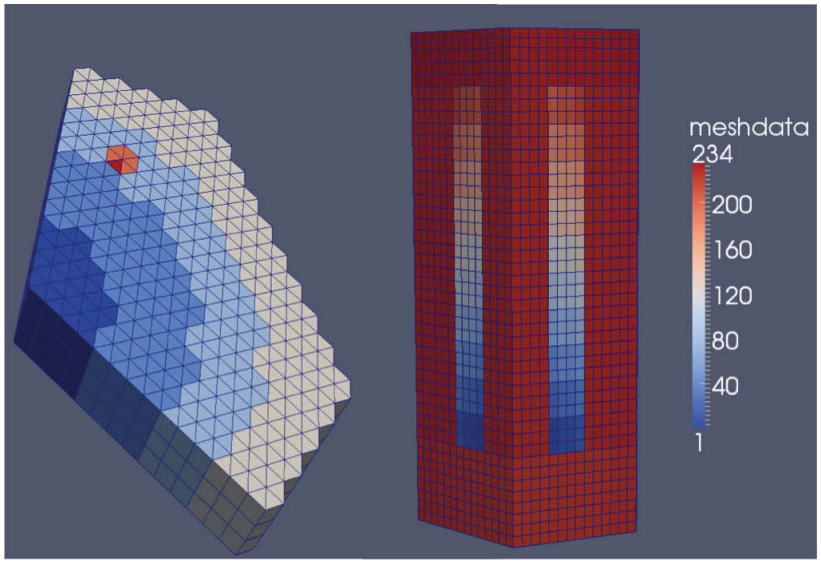

Figure 4: PRONGHORN Model of the MHTGR-350 


\section{References}

[1] D. Andrs, R. Berry, D. Gaston, R. Martineau, J. Peterson, H. Zhang, H. Zhao, and L. Zou. Relap-7 level 2 milestone report: Demonstration of a steady state single phase pwr simlulation with relap-7. Technical report, Idaho National Laboratory, 2012.

[2] H. Park, D.A. Knoll, D.R. Gaston, and R.C. Martineau. Toghly coupled multiphysics algorithms for pebble bed reactors. Nuclear Engineering and Design, 166:118-133, April 2010.

[3] Shijie Liu and Jacob H. Masliyah. Handbook of Porous Media. Taylor \& Francis, Boca Rotan, Florida, 2005.

[4] P. N. Brown and Y. Saad. Hybrid krylov methods for nonlinear systems of equations. SIAM J. Sci. Stat. Comput., 11(3):450, 1990.

[5] D.A. Knoll and D.E. Keyes. Jacobian-free newtown-krylov methods: A survey of approaches and applications. J. Comput. Phys., 193(2):357, 2004.

[6] D. Marais. Validation of the point kinetic neutronic model of the pbmr. Master's thesis, NorthWest University, 53 Borcherd Street, Potchefstroom, 2531, South Africa, May 2007.

[7] J. Ortensi. Prismatic core coupled transient benchmark. Trans. Amer. Nuc. Soc., June 2011.

[8] A. Narasimhan and J L. Lage. Modified hazen-dupuit-darcy model for forced convection of a fluid with temperature dependent viscoisty. Journal of Heat Transfer, 123(38):31-38, 2000. 


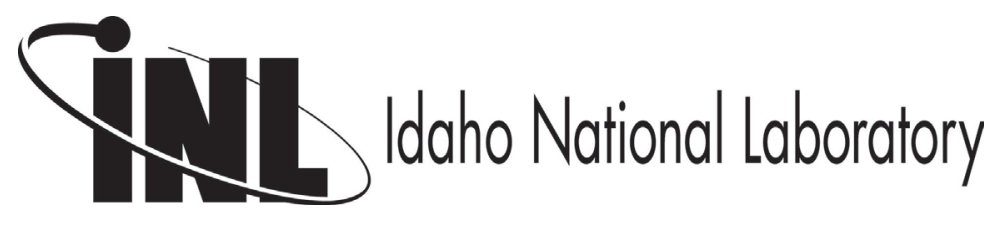

